\title{
Experience with onabotulinumtoxinA (BOTOX) in chronic refractory migraine: focus on severe attacks
}

\author{
A. Oterino $\cdot$ C. Ramón · J. Pascual
}

Received: 8 December 2010/Accepted: 9 January 2011/Published online: 5 February 2011

(C) The Author(s) 2011. This article is published with open access at Springerlink.com

\begin{abstract}
The objective of this study is to analyse our experience in the treatment of refractory chronic migraine (CM) with onabotulinumtoxinA (BTA) and specifically in its effects over disabling attacks. Patients with $\mathrm{CM}$ and inadequate response or intolerance to oral preventatives were treated with pericranial injections of $100 \mathrm{U}$ of TBA every 3 months. The dose was increased up to $200 \mathrm{U}$ in case of no response. The patients kept a headache diary. In addition, we specifically asked on the effect of BTA on the frequency of disabling attacks, consumption of triptans and visits to Emergency for the treatment of severe attacks. This series comprises a total of 35 patients ( 3 males), aged 24-68 years. All except three met IHS criteria for analgesic overuse. The number of sessions with BTA ranged from 2 to 15 (median 4) and nine (26\%) responded (reduction of $>50 \%$ in headache days). However, the frequency of severe attacks was reduced to an average of $46 \%$. Oral triptan consumption (29 patients) was reduced by $50 \%$ (from an average of 22 to 11 tablets/month). Those six patients who used subcutaneous sumatriptan reduced its consumption to a mean of $69 \%$ (from 4.5 to 1.5 injections per month). Emergency visits went from an average of 3 to 0.4 per trimester $(-83 \%)$. Six patients complained of mild adverse events, transient local cervical pain being the most common. Although our data must be taken with caution as this is an open trial, in clinical practice treatment of refractory $\mathrm{CM}$ with BTA reduces the frequency of disabling attacks, the
\end{abstract}

\footnotetext{
A. Oterino

Service of Neurology, University Hospital Marqués de

Valdecilla, Santander, Spain

C. Ramón · J. Pascual $(\varangle)$

Neuroscience Area, Service of Neurology,

University Hospital Central de Asturias, Oviedo, Spain

e-mail: juliopascual@telefonica.net
}

consumption of triptans and the need of visits to Emergency, which makes this treatment a profitable option both clinically and pharmacoeconomically.

Keywords Chronic migraine $\cdot$ Chronic refractory migraine $\cdot$ OnabotulinumtoxinA

\section{Introduction}

Chronic migraine, understood as the presence of headache during 15 or more days in a month in patients with migraine history, is an important health problem [1]. It is estimated that about $2 \%$ of general population meets the criteria for chronic migraine with or without analgesic overuse [2,3]. Prevalence in women around 40-50 years of age reaches $5 \%$, thus justifying that almost $5 \%$ of consultations to Neurology Services in Spain are caused by this condition [4].

Chronic migraine is important not only on account of its great frequency, but also because it significantly reduces the quality of life of the patients affected and it determines an unquestionable morbidity [5]. On one hand, these patients often develop complications connected with analgesic consumption, such as upper digestive haemorrhage or analgesic nephropathy [6]. On the other hand, they usually associate chronic depression, partly due to the increased frequency of disabling pain [3,5]. Lastly, recent data suggest the increased frequency of severe attacks related to chronic migraine is a stroke risk factor [7].

Migraine treatment is a complex issue. If analgesic overuse is present, especially opiates or ergotics, withdrawal becomes compulsory. Most patients are in need of a preventive treatment. Even though with the exception of topiramate $[8,9]$, there are no controlled trials in chronic 
migraine for the majority of the preventative treatments used in episodic migraine, clinical practice suggests that beta-blockers, amitryptiline, flunarizine and some neuromodulators can be useful when treating these patients. However, a relevant subgroup of these patients does not respond effectively to any of the preventative treatments. These patients met the criteria for chronic refractory migraine [10]. Aggressive options such as bilateral suboccipital stimulation have been tried lately in these patients with poor results. Recently, effectiveness of onabotulinumtoxinA (BTA), BOTOX to be precise, has been proven for preventive treatment of patients with chronic migraine [11-13]. Our goal was to assess our experience in treating those patients with chronic refractory migraine with BTA in daily clinical practice, focusing on its effect on disabling attacks and taking into account various parameters that had not been specifically studied in clinical trials.

\section{Patients and methods}

Subjects involved in this study were patients from our refractory headache clinic and had to satisfy the following requirements: (1) meet the criteria for chronic migraine with or without analgesic overuse; (2) show insufficient response (over a minimum of 6 weeks) or absence of tolerability of beta-blockers, flunarizine, topiramate, valproic acid and amitryptiline; and (3) give their informed consent to pericranial treatment with BTA. All patients with criteria for analgesic abuse had failed in at least one withdrawal attempt. Both patients with fibromyalgia and active depression as well as those overusing ergotics or opiates were excluded. Patients were allowed to continue with preventative oral medication during the treatment with BTA.

Patients were treated with BTA every 3 months. All patients received a minimum of two treatments. The first of them consisted of injecting $100 \mathrm{U}$ into 20 sites (5 units per site) distributed among the muscles of each hemicranium as follows: one site into corrugator, two into frontalis, three into temporalis, two into suboccipitalis, one into semispinalis and one into splenius. In case of insufficient response, the dose was increased up to a maximum of $200 \mathrm{U}$ and 40 sites in accordance with the protocol for phase III trials.

Patients kept a conventional headache diary regularly. Under this study we took into consideration the diary noted in the second month of the last quarter of the treatment and compared it to the one written during the pretreatment. Consequently, we compiled specific information about the effect of BTA on disabling attacks and on consumption of triptans as well as about the visits to Emergency, whether it be a health centre or the hospital, for parenteral treatment of attacks refractory to domiciliary management.

\section{Results}

Our series covers 35 patients ( 3 males) aged between 24 and 68. Only three of them did not meet the criteria for analgesic overuse. The average of headache days per month before treatment with BTA was 24.7, while that of days with severe, disabling headaches was 8.2 per month. A total of 29 patients were taking oral triptans regularly (an average of 22 pills/month before the study) and 6 were also using subcutaneous sumatriptan (an average of 4.5 injections/month before the study). Only one patient was not undergoing oral preventative treatment. At the time of the evaluation 16 patients were taking one oral preventative, 15 were taking two and 3 patients were undergoing medical tritherapy. Drugs used were in the order: topiramate, 15 patients; amitriptyline, 12; zonisamide, 7; beta-blockers, 5; valproic acid, 4; candesartan 4 and flunarizine, 4.

The number of treatments ranged between a minimum of 2 and a maximum of 16 (median 4). "Response" (reduction of headache days per month at least by 50\%) was observed in 9 patients (26\%) and "excellent response" ( $>75 \%$ reduction) in only 2 of them $(6 \%)$. The average number of days with severe, disabling headache after treatment was 3.8 per month (mean reduction $46 \%$, limits 0-905). Consumption of oral triptans in 29 patients who took them regularly halved (from 22 to 11 oral triptans/month). The 6 patients using subcutaneous sumatriptan went from 4.5 injections/month to 1.5 injections/ month (69\% reduction). Finally, the average number of visits to casualty department for parenteral treatment went from 3 in the pretreatment quarter to 0.4 (87\% reduction) (Fig. 1).

Only six patients (18\%) experienced adverse effects, always mildly and temporarily, consisting of cervical pain in four cases and eyebrow asymmetry in two of them.

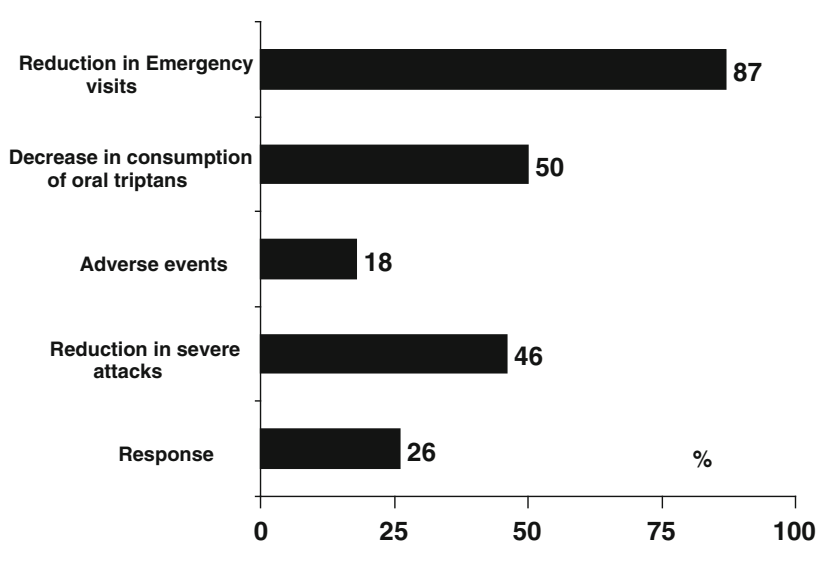

Fig. 1 Summary of the main results of this study 


\section{Discussion}

Our experience in clinical practice conditions brings effectiveness and excellent tolerability to pericranial injections of BTA for the treatment of chronic refractory migraine along the lines of published clinical trials [1113]. Like in those trials, "response" rate, i.e., the reduction of headache days at least by $50 \%$, was not outstanding. Only one-fourth of the patients met the criteria of response laid down by the International Headache Society and less than $10 \%$ fulfilled the criteria for excellent response [14]. If we analyze, however, what occurred in disabling attacks, BTA's effectiveness was very clear: their frequency halved and the number of visits to Emergency was reduced by almost $90 \%$. These data explain what seems a discrepancy in the results (in terms of response) of the clinical trials, which are not spectacular over placebo, and what happens in clinical practice, where the patient declares to feel clearly better although the number of headache days in the diary has not dropped dramatically. However, even taking into consideration that during our series patients had been refractory to various treatments, we cannot rule out a placebo effect; these data suggest that the effect of BTA in chronic migraine lies in a downward modulation of severe pain attacks, which would then be less invalidating and easier to deal with. This is endorsed by two of our results: both the halved consumption of triptans, drugs these patients only take for their most disabling attacks, and the dramatic decrease in the number of visits to Emergency for parenteral treatment. Again, however, we would like to emphasize that these data must be taken with caution as no placebo arm was included in this trial. This is not a formal pharmacoeconomic study, but its results can help to illustrate the potential cost advantages of this new treatment approach. Consider the following examples: an easy calculation taking into consideration the local average price of oral triptans indicates that the savings-only in oral triptans_-per patient and month would be of $€ 101$. Savings in casualty department visits, taking into account the official price in our country of $€ 138$ per visit without further studies, is even higher.

Our treatment protocol differs in some aspects from the one carried out in phase III of the clinical trials. In this study we administered an initial dose of $100 \mathrm{U}$ and we only raised it to 150-200 $\mathrm{U}$ in those patients whose response was insufficient. The same happened with the number of points injected, which was lower (20 compared to a minimum of 31). Same muscular groups were injected, except for the trapezius, which was left with no treatment. Positive open results for BTA with the same dose and lower than ours exist [15-17]. Therefore, these differences are logical since the protocol of the clinical trial tries to guarantee that a potential lack of effectiveness is not due to an insufficient dose, as against the daily clinical practice that tries to optimize the dose and comfort of the patient.

To conclude, we would like to highlight that BTA has been useful in our experience with patients with chronic refractory migraine who showed analgesic overuse in most cases. Although we excluded patients abusing of ergotics and opiates from the study, these results indicate, along the lines of phase II results [18-20], that patients with chronic migraine and analgesic overuse can improve specifically with preventative treatment, in this instance BTA.

Acknowledgments This study was conducted independently without any kind of support from the pharmaceutical industry. We thank Paula Pascual for her stylistic review of the manuscript.

\section{Conflict of interest None.}

Open Access This article is distributed under the terms of the Creative Commons Attribution License which permits any use, distribution and reproduction in any medium, provided the original author(s) and source are credited.

\section{References}

1. Olesen J, Bousser MG, Diener HC et al (2006) New appendix criteria open for a broader concept of chronic migraine. Cephalalgia 26:742-746

2. Castillo J, Muñoz P, Guitera V, Pascual J (1999) Epidemiology of chronic daily headache in the general population. Headache 39:190-196

3. Jensen R, Stovner LJ (2008) Epidemiology and comorbidity of headache. Lancet Neurol 7:354-361

4. Pascual J, Sánchez del Río M, Jiménez-Hernández MD et al (2010) La migraña crónica vista por el neurólogo y el paciente. Rev Neurol 50:705-710

5. Colás R, Muñoz P, Temprano R, Gómez C, Pascual J (2004) Chronic daily headache with analgesic overuse: epidemiology and impact on quality of life. Neurology 62:1338-1342

6. Giopponi S, Venturelli E, Rao R, Liberini P, Padovani A (2010) Hypertension is a factor associated with chronic daily headache. Neurol Sci 31(Suppl 1):S171-S173

7. Kruit MC, van Buchem MA, Launer LJ, Terwindt GM, Ferrari MD (2010) Migraine is associated with an increased risj of deep white matter lesions, subclinical posterior circulation infacts and brain iron accumulation: the population-based MRI CAMERA study. Cephalalgia 30:129-136

8. Diener HC, Bussone G, Van Oene JC, Lahaye M, Schwalen S, Goadsby PJ (2007) Topiramate reduces headache days in chronic migraine: a randomized, double-blind, placebo-controlled study. Cephalalgia 27:814-823

9. Silberstein SD, Lipton RB, Dodick DW (2007) Efficacy and safety of topiramate for the treatment of chronic migraine. A randomized, double-blind, placebo-controlled trial. Headache 47:170-180

10. Schulman EA, Lake AE III, Goadsby PJ et al (2008) Defining refractory migraine and refractory chronic migraine: proposed criteria from the refractory headache special interest section of the American Headache Society. Headache 48:778-782

11. Diener HC, Dodick DW, Aurora SK et al (2010) OnabotulinumtoxinA for treatment of chronic migraine: results from the 
double-blind, randomized, placebo-controlled phase of the PREEMPT 2 trial. Cephalalgia 30:804-814

12. Aurora SK, Dodick DW, Turkel CC et al (2010) OnabotulinumtoxinA for treatment of chronic migraine: results from the double-blind, randomized, placebo-controlled phase of the PREEMPT 1 trial. Cephalalgia 30:793-803

13. Dodick DW, Turkel CC, DeGryse RE et al (2010) OnabotulinumtoxinA for the treatment of chronic migraine: pooled results from the double-blind, randomized, placebo-controlled phases of the PREEMPT clinical program. Headache 50:921-936

14. Silberstein S, Tfelt-Hansen P, Dodick DW et al (2008) Guidelines for controlled trials of prophylactic treatment of chronic migraine in adults. Cephalalgia 28:484-495

15. Silberstein S, Mathew N, Saper J, Jenkins S, Group for the BOTOX Migraine Clinical Research Group (2000) Botulinum toxin type $\mathrm{A}$ as a migraine preventive treatment. Headache 40:445-450

16. Farinelli I, Coloprisco G, De Filippis S, Marteletti P (2006) Longterm benefits of botulinum toxin type A (BOTOX) in chronic daily headache: a five-year long experience. J Headache Pain 7:407-412

17. Freitag FG, Diamond S, Diamond M, Urban G (2008) Botulinum toxin type $\mathrm{A}$ in the treatement of chronic migraine without medication overuse. Headache 48:201-209

18. Mathew NT, Frishberg BM, Gawel M, Dimitrova R, Gibson J, Turkel C, the BOTOC CDH Study Group (2005) Botulinum toxin type A for the prophylaxis of chronic daily headache: a randomized, double-blind, placebo-controlled trial. Headache 45:293-307

19. Silberstein SD, Stark SR, Lucas SM, Christie SN, DeGryse ER, Turkel CC, BoNTA-039 Group (2005) Botulinum toxin type A for the prophylactic treatment of chronic daily headache: a randomized, double-blind, placebo-controlled trial. Mayo Clin Proc 80:1126-1137

20. Dodick DW, Mauskop A, Elkind AH, DeGryse R, Brin MF, Silberstein SD (2005) Botulinum toxin type A for the prophylaxis of patients not receiving other prophylactic medications: a randomized double-blind, placebo-controlled study. Headache 45:315-324 